\title{
Linearization Method of Nonlinear Magnetic Levitation System
}

\author{
Dini Wang $\mathbb{D}$, Fanwei Meng $\mathbb{D}$, and Shengya Meng \\ School of Control Engineering, Northeastern University at Qinhuangdao, Qinhuangdao 066004, China \\ Correspondence should be addressed to Fanwei Meng; mengfanwei@neuq.edu.cn
}

Received 5 May 2020; Accepted 3 June 2020; Published 22 June 2020

Guest Editor: Weicun Zhang

Copyright (c) 2020 Dini Wang et al. This is an open access article distributed under the Creative Commons Attribution License, which permits unrestricted use, distribution, and reproduction in any medium, provided the original work is properly cited.

Linearized model of the system is often used in control design. It is generally believed that we can obtain the linearized model as long as the Taylor expansion method is used for the nonlinear model. This paper points out that the Taylor expansion method is only applicable to the linearization of the original nonlinear function. If the Taylor expansion is used for the derived nonlinear equation, wrong results are often obtained. Taking the linearization model of the maglev system as an example, it is shown that the linearization should be carried out with the process of equation derivation. The model is verified by nonlinear system simulation in Simulink. The method in this paper is helpful to write the linearized equation of the control system correctly.

\section{Introduction}

Magnetic levitation generates eddy-current effect by metal objects under the action of high-frequency electromagnetic fields, which enables metal objects to be suspended stably in the air [1]. Magnetic levitation technology is developing rapidly because it essentially eliminates friction and has advantages unmatched by other traditional technologies [2]. The application of the magnetic levitation technology covers numerous fields, such as high-speed magnetic bearing [3, 4], high-speed levitation train [5-7], and wind tunnel magnetic levitation [8]. Therefore, the control design for the maglev system is particularly important.

The maglev system is a nonlinear system, which causes great difficulties in its control design [9-11]. Literature [12] proposes a model-independent control system design method based on U-model. In the linear control design method, linearization is often the first step in the control system design and modeling of the magnetic levitation system [13].

It is generally considered that the nonlinear equation is linearized as long as the Taylor expansion is used [14]. However, the Taylor expansion method only applies to the original nonlinear function. The equations for general magnetic levitation systems are usually obtained from many derivations. In the process of building the nonlinear model, the calculation of partial derivative has been done. According to the derivation algorithm, if we continue to take the derivative, then the partial derivative should be treated as a constant. In traditional modeling, this constant has been treated as a variable. And the error is introduced as we continue to differentiate. If linearization is simply carried out through the Taylor expansion method according to the derived equation, the result may be wrong.

Based on the analysis of the Lagrange equation [15], this paper points out that the partial derivative of Lagrange equation should be taken according to the operating point to get the correct linearized equation. Simulink simulation results show that the method proposed in this paper is very effective for the linearization of the magnetic levitation system and is worth popularizing.

\section{Maglev System Model and Its Linearization}

In general, the linearization of nonlinear systems is through Taylor series expansion, and a linear relation can be obtained after omitting the higher order terms [16]. Taylor series refers to the expansion of a nonlinear function $f(x, y)$. For example, formula (1) is the equation of a prime mover [17].

$$
J \frac{\mathrm{d} \omega}{\mathrm{d} t}=T_{M}(\omega, u)-T_{D}(\omega)
$$


where $T_{M}$, the driving torque, is a nonlinear function of the rotation rate $\omega$ and the control input $u$ and $T_{D}$, the load torque, is a function of the rotation rate $\omega$. If $T_{M}$ and $T_{D}$ are expanded as Taylor series and take the first term, the linearized equation of formula (1) can be obtained. This is the general notion of linearization. But for a general nonlinear system, it takes a lot of arithmetical operation to get from the original equation to the nonlinear equation. Using Taylor series to linearize the nonlinear terms in the final result, wrong result is often obtained.

The model of the typical electromagnetic suspension (EMS) train control system is shown in Figure 1.

The motion equation of the maglev system model can be written as $[11,15]$

$$
\begin{aligned}
m \frac{\mathrm{d}^{2} z(t)}{\mathrm{d} t^{2}} & =-\frac{\mu_{0} N^{2} a_{m}}{4}\left(\frac{i(t)}{z(t)}\right)^{2}+m g \\
\frac{\mathrm{d} i(t)}{\mathrm{d} t} & =\frac{i(t)}{z(t)} \frac{\mathrm{d} z(t)}{\mathrm{d} t}-\frac{2}{\mu_{0} N^{2} a_{m}} z(t)\left(R_{m} i(t)-u(t)\right),(3)
\end{aligned}
$$

where $\mu_{0}$ represents the permeability in a vacuum; $m$ represents the mass of the suspension magnet, $m=15 \mathrm{~kg} ; a_{m}$ represents the effective area of the magnetic pole, $a_{m}=1.024 \times 10^{-2} \mathrm{~m}^{2} ; N$ is the number of turns of the coil on the electromagnet, $N=280 ; R_{m}$ represents the resistance of the coil, $R_{m}=1.1 \Omega ; u(t)$ represents the control voltage at both ends of the coil; $i(t)$ represents the current in the coil; $z(t)$ is the gap between the electromagnet and the guideway; and $g$ represents the gravitational acceleration. Suppose that the state vector is

$$
x(t)=[z(t) \dot{z}(t) i(t)]^{T}=\left[x_{1} x_{2} x_{3}\right]^{T} \in R^{3} .
$$

Consider the nonlinear terms $(i(t) / z(t))^{2},(i(t) / z(t))$, $z(t) i(t)$ in (2) and (3) as nonlinear functions. The linearized equation of the system can be obtained by Taylor series expansion [16].

$$
\dot{\mathbf{x}}=\mathbf{A x}+\mathbf{B u}
$$

The corresponding matrices can be written as

$$
A=\left[\begin{array}{ccc}
0 & 1 & 0 \\
\frac{\mu_{0} a_{m} N^{2}}{2 m} \frac{i_{0}^{2}}{z_{0}^{3}} & 0 & -\frac{\mu_{0} a_{m} N^{2}}{2 m} \frac{i_{0}}{z_{0}^{2}} \\
\left(-\frac{2 R_{m}}{\mu_{0} N^{2} a_{m}}-\frac{\dot{z}_{0}}{z_{0}^{2}}\right) i_{0} & \frac{i_{0}}{z_{0}}-\frac{2 R_{m}}{\mu_{0} N^{2} a_{m}} z_{0}+\frac{\dot{z}_{0}}{z_{0}}
\end{array}\right],
$$

$$
B=\left[\begin{array}{c}
0 \\
0 \\
\frac{2}{\mu_{0} N^{2} a_{m}} z_{0}
\end{array}\right] .
$$

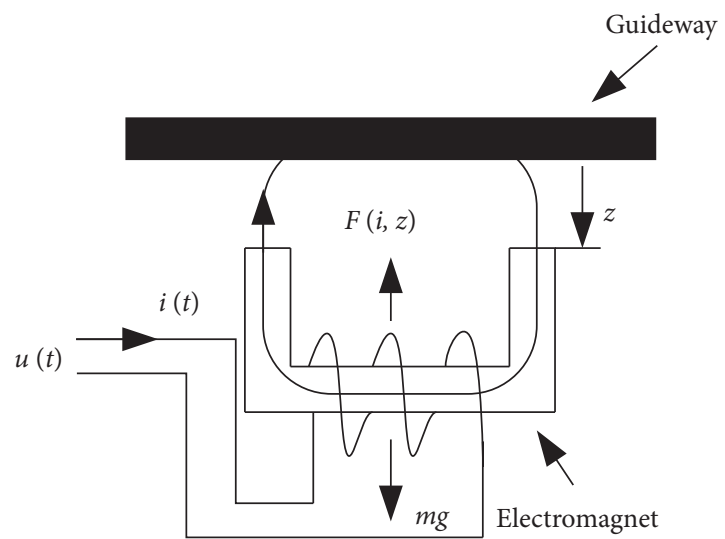

Figure 1: Model of an electromagnetic suspension system.

Set the nominal working point as $z_{0}, z_{0}=4.0 \times 10^{-3} \mathrm{~m}$, and the corresponding operating current $i_{0}=3.0538 \mathrm{~A}$. Then, the linearized equation can be written as

$$
\dot{x}=\left[\begin{array}{ccc}
0 & 1 & 0 \\
4900.1 & 0 & -6.4184 \\
-6659.4 & 763.45 & -8.7228
\end{array}\right] x+\left[\begin{array}{c}
0 \\
0 \\
7.9298
\end{array}\right] u .
$$

Formulas (5) and (7) are directly obtained by the Taylor expansion method according to formulas (2) and (3). This is a common linearization method, but it proves (see below) that the resulting linearization equation is incorrect.

Next, we start with the establishment of the equation. For the dynamic system with $k$ generalized coordinates $q_{1}, \ldots, q_{k}$, the Lagrange equation is $[2,4]$

$$
\frac{\mathrm{d}}{\mathrm{d} t} \frac{\partial T}{\partial \dot{q}_{i}}-\frac{\partial T}{\partial q_{i}}=-\frac{\partial V}{\partial q_{i}}-\frac{\partial R}{\partial \dot{q}_{i}}+f_{i}(t), \quad i=1,2 \ldots, k,
$$

where $T$ represents the kinetic energy stored by the dynamic system, $V$ represents the potential energy of the system, $f_{i}(t)$ represents the external force varying with time, and $R$ represents Rayleigh's dissipation function, and its general form can be written as

$$
R=\frac{1}{2} R_{m} \dot{q}^{2} .
$$

Suppose the generalized coordinates of the maglev control system are air gap $z$ and charge $q$ (note $\dot{q}=i$ ), then the kinetic energy and potential energy of the system are, respectively,

$$
\begin{aligned}
& T=\frac{1}{2} m \dot{z}^{2}+\frac{1}{2} L \dot{q}^{2}, \\
& V=-m g z,
\end{aligned}
$$

where

$$
L=\frac{\mu_{0} N^{2} a_{m}}{2 z} .
$$

By substituting formulas (10) (12) into Lagrange equation (8), the equation of generalized coordinates $z$ and $q$ can be written as 


$$
\begin{aligned}
\frac{\mathrm{d}}{\mathrm{d} t}(m \dot{z})-\frac{1}{2} \dot{q}^{2}\left(-\frac{\mu_{0} N^{2} a_{m}}{2 z^{2}}\right) & =m g, \\
\frac{\mathrm{d}}{\mathrm{d} t}\left(\frac{\mu_{0} N^{2} a_{m}}{2 z} \dot{q}\right) & =-R_{m} \dot{q}+u .
\end{aligned}
$$

The derivative of (14) on the left is

$$
\frac{\mathrm{d}}{\mathrm{d} t}\left(\frac{\mu_{0} N^{2} a_{m}}{2 z} \dot{q}\right)=\frac{\mu_{0} N^{2} a_{m}}{2 z} \frac{\mathrm{d} i(t)}{\mathrm{d} t}-\frac{\mu_{0} N^{2} a_{m} i}{2 z^{2}} \frac{\mathrm{d} z(t)}{\mathrm{d} t} .
$$

Thus, according to (13) and (14), the equation of motion of the system can be finally obtained as

$$
\begin{gathered}
\frac{\mathrm{d}^{2} z(t)}{\mathrm{d} t^{2}}=-\frac{\mu_{0} N^{2} a_{m}}{4 m}\left(\frac{i(t)}{z(t)}\right)^{2}+g, \\
\frac{\mathrm{d} i(t)}{\mathrm{d} t}=\frac{i(t)}{z(t)} \frac{\mathrm{d} z(t)}{\mathrm{d} t}-\frac{2}{\mu_{0} N^{2} a_{m}} z(t)\left(R_{m} i(t)-u(t)\right) .
\end{gathered}
$$

Equations (16) and (17) are equivalent to (2) and (3) when analyzing the maglev system. Since we have taken the partial derivative once when we derived (17), the partial derivative should be a constant to the operating point in linearization, that is, it corresponds to a constant coefficient instead of a variable. Therefore, if linearization is carried out, the corresponding constants of all partial derivatives in (15) should be taken, and (15) can be rewritten as

$$
\frac{\mathrm{d}}{\mathrm{d} t}\left(\frac{\mu_{0} N^{2} a_{m}}{2 z} \dot{q}\right)=\frac{\mu_{0} N^{2} a_{m}}{2 z_{0}} \frac{\mathrm{d} i(t)}{\mathrm{d} t}-\frac{\mu_{0} N^{2} a_{m} i_{0}}{2 z_{0}^{2}} \frac{\mathrm{d} z(t)}{\mathrm{d} t} .
$$

The corresponding linearized equation can be obtained by substituting (18) into $(14) .(i(t) / z(t))^{2}$ in (16) is indeed a nonlinear function, and its linearization relation can be obtained by Taylor expansion.

$$
\begin{aligned}
& \frac{\mu_{0} N^{2} a_{m}}{4 m}\left(\frac{i(t)}{z(t)}\right)^{2}=\frac{\mu_{0} N^{2} a_{m}}{4 m}\left(\frac{i_{0}}{z_{0}}\right)^{2}+\frac{\mu_{0} N^{2} a_{m}}{2 m}\left(\frac{i_{0}}{z_{0}^{2}}\right) \Delta i \\
& -\frac{\mu_{0} N^{2} a_{m}}{2 m}\left(\frac{i_{0}^{2}}{z_{0}^{3}}\right) \Delta z .
\end{aligned}
$$

In this way, by substituting (18) and (19) into (14) and (16), the final linearized equation can be obtained as

$$
\dot{x}=\left[\begin{array}{ccc}
0 & 1 & 0 \\
\frac{\mu_{0} a_{m} N^{2}}{2 m} \frac{i_{0}^{2}}{z_{0}^{3}} & 0 & -\frac{\mu_{0} a_{m} N^{2}}{2 m} \frac{i_{0}}{z_{0}^{2}} \\
0 & \frac{i_{0}}{z_{0}}-\frac{2 R_{m}}{\mu_{0} N^{2} a_{m}} z_{0}
\end{array}\right] x+\left[\begin{array}{c}
0 \\
0 \\
\frac{2}{\mu_{0} N^{2} a_{m}} z_{0}
\end{array}\right] u,
$$

$$
\dot{x}=\left[\begin{array}{ccc}
0 & 1 & 0 \\
4900.1 & 0 & -6.4184 \\
0 & 763.45 & -8.7228
\end{array}\right] x+\left[\begin{array}{c}
0 \\
0 \\
7.9298
\end{array}\right] u .
$$

Obviously, (20) and (21) are obviously inconsistent with (5) and (7). The state matrix of (7) has the element -6659.4 in the third row and first column, while the element at this position in (21) is 0 . In order to verify this linearization method, we use the nonlinear system simulation in this paper because when the deviation is very small, the nonlinear system results should be consistent with the linearization result.

\section{Simulation of Nonlinear System}

Next, we will simulate and analyze the nonlinear equations of the maglev system. In the nonlinear simulation of Simulink, a custom function module Fon is mainly used. The function of this module is to process the input by the rules conforming to $\mathrm{C}$ language. The corresponding formulas may consist of one or more components. $u$ is the input of the module, which supports vectorized input. If $u$ is a vector, $u$ [i] represents the $i$ th element of the vector, and $u$ [1] or a single $u$ represents the first element. The function module can perform both mathematical operations (such as addition, subtraction, multiplication, and division) and relational operations (such as identically equal, not equal, greater than, and equal), as well as three kinds of logical operations. The precedence of these operators conforms to the rules in $\mathrm{C}$ language.

The simulation model of the magnetic levitation system based on differential (2) and (3) is shown in Figure 2. In Figure 2, the function of Mux module is to organize several signals into one signal. Here, variables are expressed in increments so as to compare with linear results. In the figure, $f_{1}(u), f_{2}(u)$, and $f_{3}(u)$ are all custom function modules.

Adopt the state feedback $k=\left[\begin{array}{lll}k_{1} & k_{2} & k_{3}\end{array}\right]=$ [ $24843.50249 .7285-20.9242]$ in the simulation. And the feedback control signal should be elicited from $\Delta z, \Delta \dot{z}, \Delta i$. In the case of module $f_{3}(u)$, it describes the operation on the right side of formula (2).

$$
f_{3}(u)=-\frac{\mu_{0} N^{2} a_{m}}{4 m}\left(\frac{\triangle i+i_{0}}{\triangle z+z_{0}}\right)^{2}+g .
$$

The input is two variables: $\Delta z, \Delta i$. The setting of $f_{3}(u)$ is shown in Figure 3.

In Figure 3, $1.6814 *\left(10^{-5}\right)$ corresponds to the value of $\left(\mu_{0} N^{2} a_{m} / 4 m\right), 9.8$ is the value of gravitational acceleration $g$, and $u(1), u(2)$ are input signals arranged in a certain order by Mux module in front of the module $f_{3}(u)$. Here, $u(1)$ corresponds to $\Delta i$ and $u(2)$ corresponds to $\Delta z$, and $i_{0}$ and $z_{0}$ are values of corresponding nominal operating points. The values of $i_{0}$ and $z_{0}$ at the equilibrium point are calculated in advance according to the following equation:

$$
m \ddot{z}(t)=-\frac{\mu_{0} N^{2} a_{m}}{4}\left(\frac{i_{0}}{z_{0}}\right)^{2}+m g=0 .
$$

i.e., 


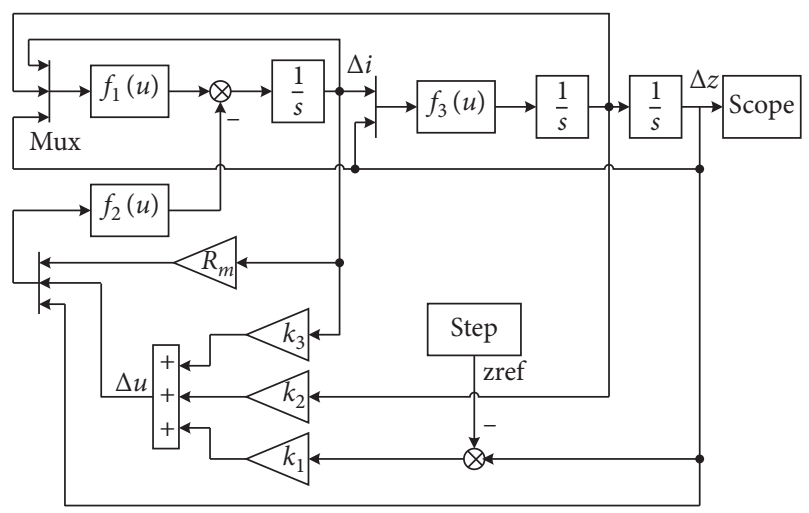

Figure 2: Simulation model of the nonlinear system.

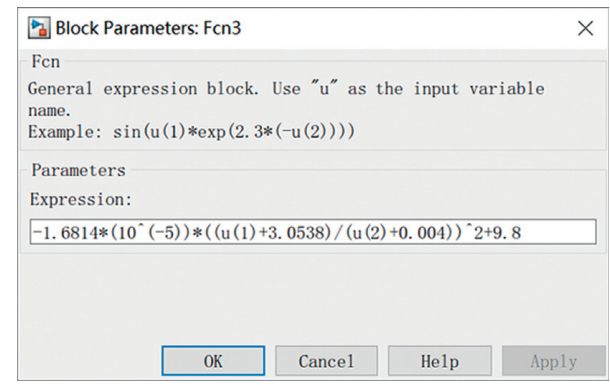

Figure 3: The dialog box of Fcn3.

The function $f_{1}(u)$ describes the first term on the right side of (3), and $f_{2}(u)$ describes the second term on the right side of (3). The problem of the nominal operating point is also set as follows:

$$
\begin{aligned}
& f_{1}(u)=\left(\frac{\triangle i+i_{0}}{\triangle z+z_{0}}\right) \Delta \dot{z}, \\
& f_{2}(u)=\frac{2}{\mu_{0} N^{2} a_{m}}\left(\triangle z+z_{0}\right)\left(R_{m} \Delta i-\Delta u\right) .
\end{aligned}
$$

\section{Verification of Linearized Equations}

Two linearization methods are proposed above. One is the simple Taylor series method ((5) and (7)), and the other is the method of taking the partial derivative as a constant when taking the column equation ((20) and (21)). In order to further verify the linearization method in this paper, the simulation checking is adopted, and the simulation results of nonlinear systems with small deviations are compared.

Figure 4 shows the simulation block diagram of the linearized system. The dotted box in the figure shows the difference between the two linearized results (7) and (21). The same state feedback is used in the simulation.

$$
k=\left[\begin{array}{lll}
k_{1} & k_{2} & k_{3}
\end{array}\right]=\left[\begin{array}{lll}
24843.50 & 249.7285 & -20.9242
\end{array}\right] .
$$

Figure 5 shows the simulation results of the step response. The short line in the figure is the simulation result of

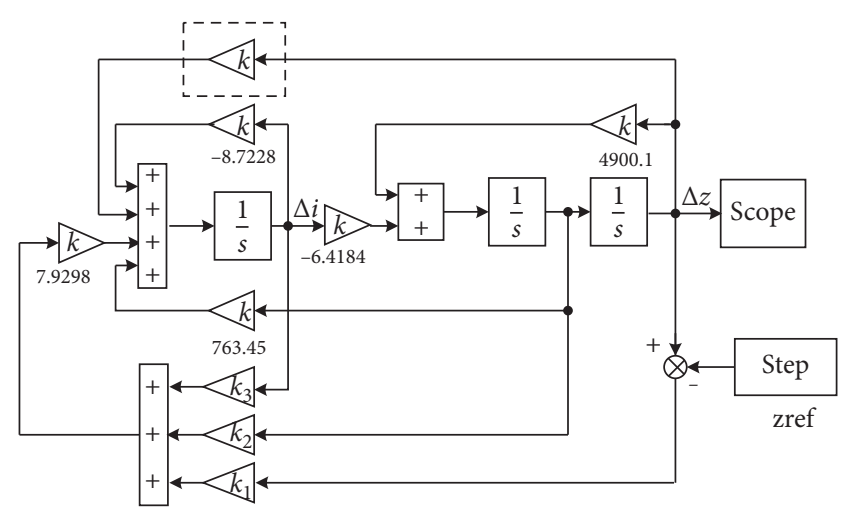

FIGURE 4: Simulation model of the linearized system.

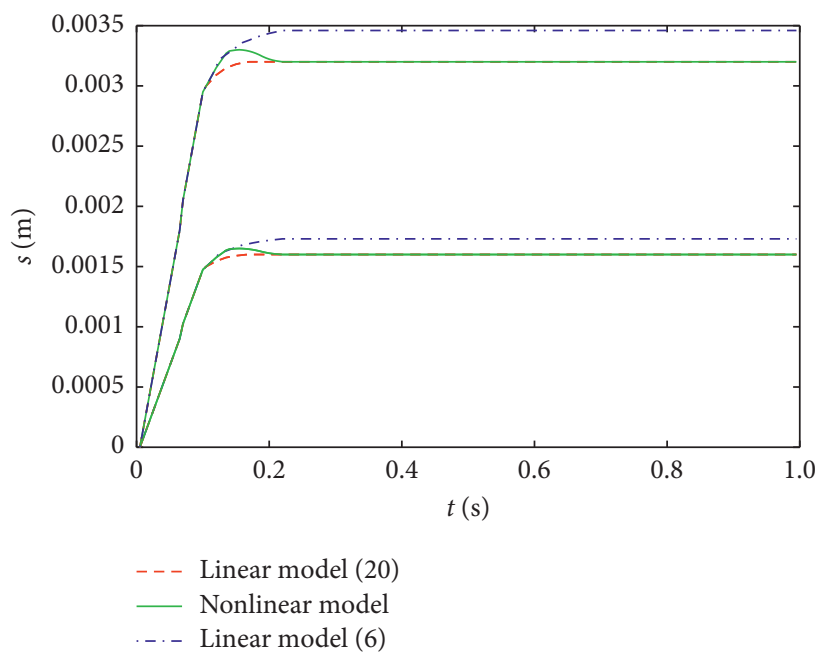

Figure 5: Comparison of the simulation results.

system (7), and the dotted line is the simulation result of system (21) of this method.

The simulation block diagram of the nonlinear system is shown in Figure 2. The solid line in Figure 5 is the simulation result of the nonlinear system, and the amplitude of the input step signal is $0.1 \mathrm{~mm}, 0.5 \mathrm{~mm}$, and $0.1 \mathrm{~mm}$, respectively. When a small signal $(0.1 \mathrm{~mm})$ is input, the dotted line of the system (21) is basically coincident with the solid line of the nonlinear system. The simulation result of system (7) is obviously inconsistent with the nonlinear result under small signals, especially the static differences are not equal. In this case, the static error of the nonlinear system is relatively easy to calculate. The static error between the system output and the step input is also shown in Figure 5 (the calculation process of the static error is abbreviated). The simulation results show that the static error of the system (21) is consistent with that of the nonlinear system.

\section{Conclusions}

The linearization of nonlinear magnetic levitation cannot be carried out simply by Taylor expansion according to the derived nonlinear equation. In the process of derivation, the 
partial derivative is included in the equation, and the linearized equation can be obtained by taking the partial derivative at the operating point. The method is simple and the physical concept is clear. On the contrary, if we linearize the equation by the Taylor series based on the derived nonlinear equation, wrong result may be obtained. In this paper, the simulation method of the nonlinear magnetic levitation system is presented, which separates the operating point (setting) from the incremental change, and is worthy of promoting.

\section{Data Availability}

The data used to support the findings of this study are available from the corresponding author upon request.

\section{Conflicts of Interest}

The authors declare that there are no conflicts of interest regarding the publication of this paper.

\section{Acknowledgments}

This study was supported in part by the Fundamental Research Funds for the Central Universities under grant no. N182304010, in part by the Natural Science Foundation of Liaoning Province under grant no. 20170520333, and in part by the Natural Science Foundation of Hebei Province under grant no. F2019501012.

\section{References}

[1] B. Gauthier-Manuel and L. Garnier, "Development of a magnetic levitation force microscope," Surface \& Interface Analysis, vol. 27, no. 5-6, pp. 287-290, 1999.

[2] K. Glatzel, G. Khurdok, and D. Rogg, "The development of the magnetically suspended transportation system in the Federal Republic of Germany," IEEE Transactions on Vehicular Technology, vol. 29, no. 1, pp. 3-17, 1980.

[3] K.-Y. Kai-Yew Lum, V. T. Coppola, and D. S. Bernstein, "Adaptive autocentering control for an active magnetic bearing supporting a rotor with unknown mass imbalance," IEEE Transactions on Control Systems Technology, vol. 4, no. 5, pp. 587-597, 1996.

[4] T. Zhang, X. Ye, L. Mo et al., "Modeling and performance analysis on the five degrees of freedom slice hybrid magnetic bearing," IEEE Transactions on Applied Superconductivity, vol. 29, no. 2, pp. 1-5, 2019.

[5] M. Zhai, Z. Long, and X. Li, "Fault-tolerant control of magnetic levitation system based on state observer in high speed maglev train," IEEE Access, vol. 7, pp. 31624-31633, 2019.

[6] X. Yang, Z. Lin, J. Ding, and Z. Long, "Lifetime prediction of IGBT modules in suspension choppers of medium/low-speed maglev train using an energy-based approach," IEEE Transactions on Power Electronics, vol. 34, no. 1, pp. 738-747, 2019.

[7] M. Komori, A. Minoda, K. Nemoto, and K. Asami, "Maximum limit of superconducting persistent current for superconducting magnetic suspension system," IEEE Transactions on Magnetics, vol. 53, no. 11, pp. 1-4, 2017.
[8] W. G. Hurley and W. H. Wolfle, "Electromagnetic design of a magnetic suspension system," IEEE Transactions on Education, vol. 40, no. 2, pp. 124-130, 1997.

[9] C. Truesdell and W. Noll, The Non-linear Field Theories of Mechanics, Springer, Berlin, Germany, 1992.

[10] A. Pang, Z. He, M. Zhao, G. Wang, Q. Wu, and Z. Li, "Sum of squares approach for nonlinear Ho control," Complexity, vol. 2018, Article ID 8325609, 7 pages, 2018.

[11] F. Meng, A. Pang, X. Dong, C. Han, and X. Sha, "Ho optimal performance design of an unstable plant under bode integral constraint," Complexity, vol. 2018, Article ID 4942906, 10 pages, 2018.

[12] Q. Zhu, L. Liu, W. Zhang, and S. Li, "Control of complex nonlinear dynamic rational systems," Complexity, vol. 2018, Article ID 8953035, 12 pages, 2018.

[13] W. T. Baumann, "Feedback control of multiinput nonlinear systems by extended linearization," IEEE Transactions on Automatic Control, vol. 33, no. 2, pp. 193-197, 1988.

[14] L. Zhu and C. R. Knospe, "Modeling of nonlaminated electromagnetic suspension systems," IEEE/ASME Transactions on Mechatronics, vol. 15, no. 1, pp. 59-69, 2010.

[15] B. V. Leer, "Towards the ultimate conservative difference scheme I. The quest of monotonicity," Proceedings of the Third International Conference on Numerical Methods in Fluid Mechanics, Springer, vol. 18, no. 1, , pp. 163-168, Berlin, Germany, 1973.

[16] Q. M. Zhu and L. Z. Guo, "A pole placement controller for non-linear dynamic plants," Proceedings of the Institution of Mechanical Engineers, Part I: Journal of Systems and Control Engineering, vol. 216, no. 6, pp. 467-476, 2002.

[17] K. Gustafson, "The original motivation: operator semigroups," Antieigenvalue Analysis: with Applications to Numerical Analysis, Wavelets, Statistics, Quantum Mechanics, Finance and Optimization, World Scientific, Singapore, 2011. 